\title{
Immunogenic Properties of the DNA Construct Encoding the Receptor-Binding Domain of the SARS-CoV-2 Spike Protein
}

\author{
M. B. Borgoyakova ${ }^{a, ~ *, ~ L . ~ I . ~ K a r p e n k o ~}{ }^{a}$, A. P. Rudometov ${ }^{a}$, D. V. Shanshin ${ }^{a}$, A. A. Isaeva ${ }^{a, b}$, \\ V. S. Nesmeyanova ${ }^{a, b}$, N. V. Volkova ${ }^{a}$, S. V. Belenkaya ${ }^{a}$, D. E. Murashkin ${ }^{a}$, \\ D. N. Shcherbakov ${ }^{a}, b$, E. A. Volosnikova ${ }^{a}$, E. V. Starostina ${ }^{a}$, L. A. Orlova ${ }^{a}$, \\ N. V. Danilchenko ${ }^{a}$, A. V. Zaikovskaya ${ }^{a}$, O. V. Pyankov ${ }^{a}$, and A. A. Ilyichev ${ }^{a}$ \\ ${ }^{a}$ Vector State Research Center of Virology and Biotechnology, Russian Federal State Agency for Health \\ and Consumer Rights Surveillance, Koltsovo, Novosibirsk oblast, 630559 Russia \\ ${ }^{b}$ World-Class Genomic Research Center for Biological Safety and Technological Independence, \\ Federal Scientific and Technical Program for the Development of Genetic Technologies, \\ Vector State Research Center of Virology and Biotechnology, Russian Federal State Agency \\ for Health and Consumer Rights Surveillance, Koltsovo, Novosibirsk oblast, 630559 Russia \\ *e-mail: borgoyakova_mb@vector.nsc.ru \\ Received March 7, 2021; revised April 19, 2021; accepted April 20, 2021
}

\begin{abstract}
The development of preventive vaccines became the first order task in the COVID-19 pandemic caused by SARS-CoV-2. This paper reports the construction of the pVAX-RBD plasmid containing the Receptor-Binding Domain (RBD) of the S protein and a unique signal sequence 176 which promotes target protein secretion into the extracellular space thereby increasing the efficiency of humoral immune response activation. A polyglucine-spermidine conjugate (PGS) was used to deliver pVAX-RBD into the cells. The comparative immunogenicity study of the naked pVAX-RBD and pVAX-RBD enclosed in the PGS envelope showed that the latter was more efficient in inducing an immune response in the immunized mice. In particular, RBD-specific antibody titers were shown in ELISA to be no higher than 1:1000 in the animals from the pVAX-RBD group and $1: 42000$, in the pVAX-RBD-PGS group. The pVAX-RBD-PGS construct effectively induced cellular immune response. Using ELISpot, it has been demonstrated that splenocytes obtained from the immunized animals effectively produced INF- $\gamma$ in response to stimulation with the S proteinderived peptide pool. The results suggest that the polyglucine-spermidine conjugate-enveloped pVAX-RBD construct may be considered as a promising DNA vaccine against COVID-19.
\end{abstract}

Keywords: SARS-CoV-2, DNA vaccine, immune response, mouse model

DOI: $10.1134 / \mathrm{S} 0026893321050046$

All currently known platforms used for vaccine development have been used to produce vaccines against Severe Acute Respiratory Syndrome Coronavirus-2 (SARS-CoV-2) including inactivated virus [1, 2], recombinant proteins and synthetic peptides [3-5], vector vaccines [6, 7], DNA-based constructs [8, 9], and RNA-based constructs [10, 11]. Each has its advantages and disadvantages. DNA vaccines are easy and quick to develop, their production costs are quite low, they are safe, and capable of inducing both humoral and cellular immunity. Although the first generations of DNA vaccines showed relatively low efficiency in inducing the immune response, the development of this platform continued with efforts

Abbreviations: COVID-19, coronavirus infectious disease 2019; PGS, polyglucine-spermidine conjugate; RBD, receptor-binding domain of the spike protein; SARS-CoV-2, severe acute respiratory syndrome coronavirus-2. being focused on the search for delivery means and adjuvants which would improve the efficiency of DNA vaccines. According to WHO, as of June 2021, 10 DNA anti-SARS-CoV-2 vaccines are undergoing clinical trials with 16 more undergoing preclinical trials (https://www.who.int/publications/m/item/draftlandscape-of-covid-19-candidate-vaccines).

The key targets of the anti-SARS-CoV-2 vaccines under development are the spike protein $(\mathrm{S})$ and its Receptor-Binding Domain (RBD) which binds with the angiotensin-converting enzyme-2 (ACE2) on the target cell surface thereby inducing viral entry [12]. Several research groups, which already have functional vector plasmids in their arsenal, were successful in quickly developing prototype vaccines and testing their immunogenicity on animals just several weeks after the SARS-CoV-2 $\mathrm{S}$ protein gene nucleotide sequence had become publicly available [8]. 
We have constructed a candidate vaccine DNA encoding the SARS-CoV-2 S protein RBD (pVAXRBD). We have optimized the codon composition of the target gene in order to increase the production level of the immunogenic protein encoded by this plasmid. A nucleotide sequence encoding the original leader sequence 176, a hybrid of the signal sequences of the two actively secreted proteins, namely, luciferase and fibroin, was attached to pVAX-RBD to ensure its efficient transport outside the cell. The polycationic polyglucine-spermidine conjugate (further referred to as PGS), which we previously used to develop the vaccines against HIV-1 [13, 14] and Ebola virus [15], and the mRNA vaccine against SARS$\mathrm{CoV}-2$ [16], was used to package and deliver the DNA vaccine. Here, we report the results of the immunogenicity study of the PGS-enveloped pVAX-RBD plasmid as a potential DNA vaccine against SARS-CoV-2.

\section{EXPERIMENTAL}

Immunogene construction. The nucleotide sequence of the gene encoding the full-size SARSCoV-2 S protein (GenBank Acc. No. MN908947) was used to construct the immunogenes. The GeneOptimizer software (https://www.thermofisher.com/ru/en/home/ life-science/cloning/gene-synthesis/geneart-gene-synthesis/geneoptimizer.html) was used to optimize the codon composition and RNA secondary structure for efficient expression in mammalian cells. The obtained nucleotide sequence was synthesized by OOO DNAsintez (Russia) and further cloned as a part of the pGH vector.

The primer pair 5'-TAATACGACTCACTATAGGCTAGCCT-3' (forward) and 5'-AAAAAAGCGGCCGCTCATTAGTTGAAGTTCACGCATTTGTTCTTC-3' (reverse) was used to amplify the S protein RBD-coding fragment $\left({ }^{320} \mathrm{~V}-{ }^{542} \mathrm{~N}\right)$ and the signal sequence 176 (MMRTLILAVLLVYFCATVHC) at the 5 '-end using the template containing the corresponding fragment (kindly provided by the Immunochemistry Laboratory of the Vector State Research Center of Virology and Biotechnology). The amplification product was inserted into the pVAX vector (ThermoFisher Scientific, United States) at the NheI and NotI restriction sites. The structure of the obtained construct was verified by Sanger sequencing in the Genomics Center for Collective Use (Russia). The obtained construct was designated pVAX-RBD.

Plasmid DNA production for immunization. Escherichia coli Stbl3 cells were chemically (calcium chloride) transformed with the pVAX and pVAX-RBD plasmids. To produce plasmid DNA for immunization, bacterial cells were grown in $2.7 \mathrm{~L}$ of LB medium with kanamycin $(25 \mu \mathrm{g} / \mathrm{mL})$. Plasmid DNA was isolated and purified using the EndoFree Plasmid Giga Kit (QIAGEN, Germany) according to the manufacturer's recommendations. Endotoxin level was determined in the LAL assay according to the manufac- turer's protocol, while gel formation was visually assessed (CharlesRiver, United States).

HEK-293T and CHO-K1 cell transfection with the DNA constructs. The HEK-293T and CHO-K1 cell cultures were obtained from the Cell Culture Collection of the Vector State Research Center of Virology and Biotechnology, Russian Federal State Agency for Health and Consumer Rights Surveillance. Lipofectamine 3000 (Invitrogen, United States) was used to transfect HEK-293T and CHO-K1 cells according to the manufacturer's instructions. $10^{7}$ cells per well were inoculated into 24-well plates and cultured in DMEM medium with $10 \%$ fetal bovine serum (FBS). On the day of transfection, the medium was substituted with maintenance medium containing $2 \%$ FBS. $2 \mu \mathrm{g}$ of plasmid DNA combined with Lipofectamine 3000 was added to the cells, and cells were incubated in $5 \% \mathrm{CO}_{2}$. After $48 \mathrm{~h}$ of incubation, cells in the culturing medium were transferred into tubes and centrifuged for $5 \mathrm{~min}$ at $800 \times g$. Then, RBD gene expression was assessed in the cell pellet and supernatant by RT-PCR and immunoblotting.

Target protein expression assessment by immunoblotting. The $\mathrm{CHO}-\mathrm{K} 1$ cell pellet was resuspended in PBS, and cells were lyzed by ultrasonication using the Soniprep 150 Plus homogenizer (MSE, Great Britain). To separate proteins, $10 \mu \mathrm{L}$ of lyzed CHO-K1 cells transfected with the pVAX-RBD or pVAX plasmid, or $10 \mu \mathrm{L}$ of supernatant (medium remaining after cell culturing) were applied to the wells of the $12 \%$ PAAG and electrophoresis was performed with the subsequent transfer of separated proteins onto the nitrocellulose membrane. The membrane was blocked with BSA, washed, and incubated in the presence of the 5000 -fold diluted hyperimmune mouse serum (serum was obtained from the blood of the mice immunized with inactivated SARS-CoV-2 (unpublished data)). Alkaline phosphatase-conjugated rabbit anti-mouse IgG antibodies were used as the secondary antibodies (Sigma, United States) with BCIP (5-bromo-4-chloro3-indolyl phosphate) and NBT (nitro blue tetrazolium) as the substrates (ThermoFisher Scientific).

RT-PCR. RBD mRNA expression level was assessed by RT-PCR. Total RNA was isolated from cells transfected with pVAX-RBD or pVAX using the RNA Isolation kit (OOO Biolabmix, Russia) and used to synthesize first-strand cDNA with subsequent PCR using the RT-PCR kit (OOO Biolabmix) and the specific primers to the RBD gene: 5'-TAATACGACTCACTATAGGCTAGCCT-3' (forward) and 5'-AAAAAAGCGGCCGCTCATTAGTTGAAGTTCACGCATTTGTTCTTC-3' (reverse). RT-PCR conditions were as follows: reverse transcription, $30 \mathrm{~min}$ at $42^{\circ} \mathrm{C}$, initial denaturation, $5 \mathrm{~min}$ at $95^{\circ} \mathrm{C}, 30$ cycles consisting of denaturation $\left(15 \mathrm{~s}\right.$ at $\left.95^{\circ} \mathrm{C}\right)$, primer annealing $\left(20 \mathrm{~s}\right.$ at $\left.58^{\circ} \mathrm{C}\right)$, and elongation $\left(1 \mathrm{~min}\right.$ at $\left.72^{\circ} \mathrm{C}\right)$, and final elongation for $5 \mathrm{~min}$ at $72^{\circ} \mathrm{C}$. Amplification 
products were visualized using electrophoresis in $1 \%$ agarose gel.

Production of the DNA-PGS complexes. At the first stage, dextran was activated via glycol group oxidation. Towards this end, 1 mol of dextran 40000 (MP Biomedicals $^{\mathrm{TM}}$, United States) was treated with 40 mol of sodium periodate for $60 \mathrm{~min}$, and the oxidized product was purified from the remaining oxidizer using the G-25 Sefadex column in $50 \mathrm{mM}$ carbonate buffer (pH 8.6). Further, spermidine (Sigma) solution was added to the oxidized dextran solution in the ratio of 15 mol of spermidine to $1 \mathrm{~mol}$ of dextran, the mixture was incubated for $2 \mathrm{~h}$, and then, sodium borohydride was added in the ratio of $80 \mathrm{~mol}$ to $1 \mathrm{~mol}$ of dextran. The mixture was incubated for 2 more hours and the target product was purified from the unreacted components by gel-filtration in PBS-equilibrated G-25 Sefadex. To form the DNA-PGS complexes, DNA was mixed with PGS in a $1: 10$ mass ratio $[15,16]$, and the product yield was assessed by the changes in the DNA electrophoretic mobility in $1 \%$ agarose gel.

Mouse immunization. Female BALB/c mice of weight 16-18 $\mathrm{g}$ were randomly divided into four groups of six mice each. Each group was immunized three times with two-week intervals using one of the following preparations: pVAX-RBD-PGS (pVAXRBD-PGS group), pVAX-RBD (pVAX-RBD group), and pVAX-PGS plasmid (PGS-enveloped pVAX plasmid; pVAX-PGS group). A solution containing $100 \mu \mathrm{g}$ of the corresponding DNA in $200 \mu \mathrm{L}$ of physiological solution was administered to the animals intramuscularly into the hind legs (100 $\mu \mathrm{L}$ into each leg).

Serum analysis using ELISA. Vaccine -induced antibody levels were assessed in the blood serum of animals six weeks after the initial immunization. Blood cellular elements were separated from sera by centrifugation $(9000 \mathrm{~g}, 15 \mathrm{~min})$ and the supernatant was heated at $56^{\circ} \mathrm{C}$ for $30 \mathrm{~min}$ to inactivate the complement system.

The RBD protein obtained in eukaryotic producer cells (CHO-K1 cells) and purified by the affinity and ion-exchange chromatography (protein purity higher that 98\%) was used as an antigen in ELISA (the protein was kindly provided by the Immunochemistry Laboratory of the Vector State Research Center of Virology and Biotechnology, Russian Federal State Agency for Health and Consumer Rights Surveillance). RBD protein $(1 \mu \mathrm{g} / \mathrm{mL}$ in $2 \mathrm{M}$ urea $)$ was immobilized on 96-well plates (Greiner Bio-One, Germany) at $4{ }^{\circ} \mathrm{C}$ overnight. Plates were washed with PBS containing $0.05 \%$ Tween 20 (PBST) and blocked with $1 \%$ casein in the same buffer for $60 \mathrm{~min}$ at room temperature. Then, serial three fold sera dilutions in the blocking solution were added to the wells starting at $1: 50$ dilution, and incubated for $60 \mathrm{~min}$ at room temperature. Plates were washed, horseradish peroxidase-conjugated rabbit anti-mouse IgG antibodies (Sigma) were added to the wells, and the plates were incubated for
$60 \mathrm{~min}$ at room temperature. TMB substrate (3,3',5,5'-tetramethylbenzidine) (Amresco, United States) was added to the wells pre-washed with PBST. Optical density at $450 \mathrm{~nm}$ was measured by the ChroMate-4300 Microplate Reader (Awareness Technology Inc., United States). Sera dilution at which optical density was more than two times higher than that in the negative control (blocking buffer was added to the wells instead of serum) was considered to be the ELISA titer.

Assessment of the virus neutralization activity of sera. The $\mathrm{nCoV} /$ Victoria/1/2020 SARS-CoV-2 strain (State Collection of Viral and Rickettsial Disease Pathogens, Vector State Research Center of Virology and Biotechnology, Russian Federal State Agency for Health and Consumer Rights Surveillance) was used in this work. A virus pool with the titer of $6.5 \operatorname{logTCID} \mathrm{D}_{50} / \mathrm{mL}$ (50\% Tissue Culture Infectious Dose) was obtained in Vero E6 cells (Cell Culture Collection of the Vector State Research Center of Virology and Biotechnology, Russian Federal State Agency for Health and Consumer Rights Surveillance). The virus working dose was $100 \mathrm{TCID}_{50}$. To assess the efficiency of the virus neutralization activity of sera, serial two fold dilutions were prepared in the range from $1: 10$ to $1: 2560$. DMEM medium containing $L$-glutamine and antibiotics $(100 \mathrm{U} / \mathrm{mL}$ of penicillin and $100 \mu \mathrm{g} / \mathrm{mL}$ of streptomycin) was used to dilute sera. Equal volumes of diluted sera samples and virus suspension containing virus working dose were mixed, incubated for $1 \mathrm{~h}$ at room temperature, then, added to the wells of the 96-well plates covered with Vero E6 cell monolayer and incubated for 4 days at $37^{\circ} \mathrm{C}$ with $5 \% \mathrm{CO}_{2}$. To stain cells, $150 \mu \mathrm{L}$ of $0.2 \%$ gentian violet solution $(1 \mathrm{~g}$ of gentian violet was added to the mixture of $20 \mathrm{~mL}$ of $96 \%$ ethanol, $120 \mathrm{~mL}$ of $40 \%$ formaldehyde, and $350 \mathrm{~mL}$ of Hank's solution). After incubating for $30 \mathrm{~min}$, liquid was discarded and the wells were washed with water. The results were visually examined. Any specific cell culture damage observed in the well was assumed to be a cytopathic effect of the virus. Neutralization activity of the immunizied animal sera was estimated based on the titer (sera dilution) at which cells were protected from the viral cytopathological activity in $50 \%$ of wells. Neutralizing antibody titers were calculated according to the Reed-Muench formula [17].

Study of the T-cell immune response. T-cell immune response level was estimated by ELISpot technique using the Mouse IFN-gamma ELISpot kit (BD, United States). Spleens were isolated from the animals two weeks after the third immunization. Splenocytes were obtained by the successive homogenizations using 70 and $40 \mu \mathrm{m}$ filters (BD Falcon, United States). After lyzing erythrocytes with the Lyzing buffer (Sigma), splenocytes were twice washed with RPMI medium and placed into $1 \mathrm{~mL}$ of RPMI medium with $2 \mathrm{mM} L$-glutamine and $50 \mu \mathrm{g} / \mathrm{mL}$ of 
Table 1. List of SARS-CoV-2 S protein peptides used to stimulate the splenocytes of BALB/c mice when studying the T-cell response using ELISpot

\begin{tabular}{r|l|l}
\hline No. & \multicolumn{1}{|c}{ Peptide } & \multicolumn{1}{|c}{ MHC restriction } \\
\hline 1 & SGTNGTKRF & H-2-Dd \\
2 & YYHKNNKSW & H-2-Kd \\
3 & KYNENGTIT & H-2-Kd \\
4 & VYAWNRKRI & H-2-Kd \\
5 & FERDISTEI & H-2-Ld \\
6 & CGPKKSTNL & \\
7 & SYQTQTNSP & H-2-Kd \\
8 & SKPSKRSFI & H-2-Dd \\
9 & KYFKNHTSP & H-2-Kd \\
10 & YPDKVFRSSVLHSTQ & H2-IEd \\
11 & DPFLGVYYHKNNKSW & H2-IEd \\
12 & KNIDGYFKIYSKHTP & H2-IEd \\
13 & RFASVYAWNRKRISN & H2-IEd, H2-IAd \\
14 & VGGNYNYLYRLFRKS & H2-IEd \\
15 & GGNYNYLYRLFRKSN & H2-IEd \\
16 & YNYKLPDDFTGCVIA & H2-IEd \\
17 & NATRFASVYAWNRKR & H2-IEd, H2-IAd \\
18 & SNGTHWFVTQRNFYE & H2-IEd \\
19 & YEQYIKWPSGRLVPR & H2-IEd \\
20 & KNKCVNFNFNGLTGT & H2-IEd \\
\hline
\end{tabular}

hentamycine. The 96-well plates provided with the kit were coated with antibodies against mouse interferon- $\gamma$ (IFN- $\gamma$ ). After the plates were blocked with RPMI, splenocytes were applied to the wells at $5 \times 10^{5}$ cells/well and stimulated with the peptide mixture containing $20 \mu \mathrm{g} / \mathrm{mL}$ of each peptide. Cells were then incubated for $20 \mathrm{~h}$ at $37^{\circ} \mathrm{C}$ with $5 \% \mathrm{CO}_{2}$, washed, and biotinylated anti-mouse IFN- $\gamma$ antibodies were added, and antibody specific binding was visualized using the streptavidin-horseradish peroxidase conjugate and the AEC (3-amino-9-ethylcarbazole) substrate. The number of IFN- $\gamma$-producing cells was calculated with the aid of the ELISpot Reader (CarlZeiss, Germany).

To stimulate the splenocytes isolated from the immunized animals, a pool of 20 SARS-CoV-2 S protein peptides recognized by the Main Histocompatibility Complex (MHC) class I (H-2-Dd, H-2-Kd, $\mathrm{H}-2-\mathrm{Ld}$ ) and class II (H2-IAd, H2-IEd) molecules from the BALB/c mice (Table 1) was used. The peptides were synthesized by AtaGenix Laboratories (China), peptide purity was more than $80 \%$.

Statistical data processing. The obtained results were statistically processed using the non-parametric Mann-Whitney test with the aid of the GraphPadPrism 6.0 software. Differences between the groups were considered significant at $p<0.05$.

\section{RESULTS}

Construct Design and In Vitro Testing of Their Properties

The nucleotide sequence used in this work corresponds to the one available in GenBank for the SARSCoV-2 S protein (MN908947). To increase the expression level and enhance the immunogenic properties of the designed DNA construct, the codon composition of the nucleotide sequence encoding SARS-CoV-2 S protein was optimized using the GeneOptimizer software. The optimized S protein-coding DNA sequence was synthesized and cloned into the pGh plasmid vector. The RBD-coding sequence and signal sequence 176 were inserted into the pVAX vector at the NheI and NotI restriction sites (Fig. 1) under the control of the human cytomegalovirus (CMV) immediate-early promoter.

As a result, the pVAX-RBD DNA construct encoding RBD along with the signal sequence was obtained. The experimental DNA vaccine was produced in a sufficient quantity and purified. Endotoxin content in the final preparation was not higher than 4 units (EU) per a single dose (100 $\mu \mathrm{g}$ DNA), which is lower than the threshold level accepted for vaccine preparations [18].

\section{RBD-Coding Sequence Expression in the HEK-293T Cells}

Expression of the RBD-coding nucleotide sequence in the HEK-293T cells was assessed by RT-PCR. Towards this end, cells were transfected with the pVAX-RBD and pVAX plasmids, total RNA was isolated, and cDNA for the RBD-coding fragment was obtained using the specific primer pair. The size of the amplified fragment corresponds to that of the RBD-coding sequence fragment (750 bp) (Fig. 2). The efficiency of HEK-293T transfection with the studied plasmids was assessed by the presence of the RBD mRNA in the cells.

\section{RBD Expression in Transfected $\mathrm{CHO}-\mathrm{K} 1$ cells}

$\mathrm{RBD}$ expression in $\mathrm{CHO}-\mathrm{K} 1$ cells was assessed by immunoblotting (Fig. 3). The presence of protein bands with molecular weight corresponding to that of RBD $(\sim 35 \mathrm{kDa})$ and binding with the antibodies obtained from the mice immunized with SARS-CoV-2 can be observed in lanes 2 and 5. Hence, the target protein is present in the transfected cells (lane 2) and is secreted by the cells to the cultural medium (lane 5). It should be noted that the protein band size in the lysate sample being slightly higher that $25 \mathrm{kDa}$ (lane 2) may be a result of incomplete glycosylation/no glycosylation of the protein product (unglycosylated protein weight is $27 \mathrm{kDa}$ ). No proteins specifically reacting with the antibodies obtained from the mice immunized with SARS-CoV-2 were observed in the cell lysate and culture medium obtained from the CHO-K1 cells transfected with the control pVAX plasmid (lanes 3 and 4). 


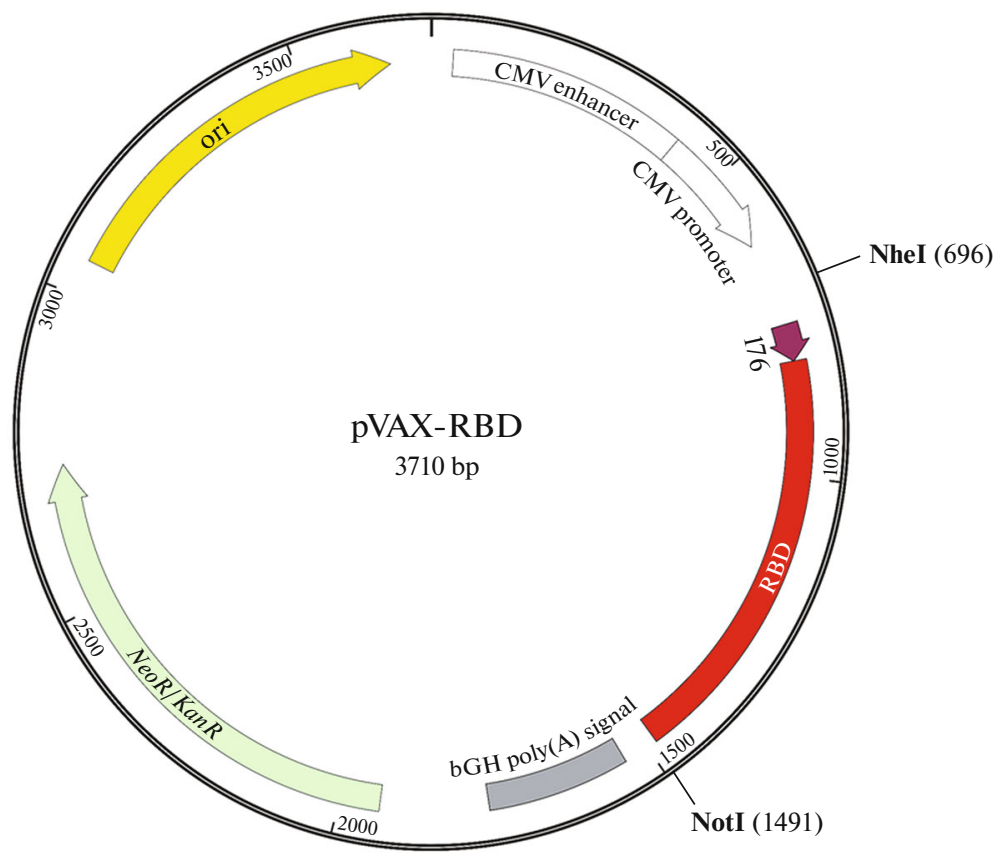

Fig. 1. pVAX-RBD plasmid genetic map. Legend: ori-replication start point; bGH poly(A) (bovine growth hormone polyadenylation) - terminal sequence enabling protein expression in eukaryotic cells, and NeoR/Kan $R$-neomycin/kanamycin resistance gene.

Thus, the RBD-coding gene is expressed at both the RNA and protein levels in the CHO-K1 cell line transfected with the pVAX-RBD construct.

\section{DNA-PGS Complex Production}

The optimal weight ratio between the DNA-polymer complex components was chosen based on the previously obtained results of the complex formation degree analysis [15]. DNA : PGS weight ratio was $1: 10$. Interaction between DNA and PGS conjugate was assessed by the electrophoretic mobility shift in the agarose gel (Fig. 4). The same weight ratio was used to form the control pVAX plasmid : PGS conjugate complex.

\section{pVAX-RBD Induces Humoral Response in Mice}

To assess the immunogenicity of the pVAXRBD-PGS and pVAX-RBD constructs, mice were immunized three times on days 0,14 , and 28 , and six weeks after the start of the experiment blood was collected from the animals for analysis (Fig. 5a). The obtained blood sera were tested for the presence of RBD-specific antibodies by means of ELISA and SARS-CoV-2 neutralization assay. The blood serum of mice immunized with the pVAX-PGS complex was used as a control.

ELISA results demonstrated that two weeks after the third immunization the average specific antibody titers in the animals who received the pVAX-
RBD-PGS construct were about 40 times higher than in the animals who received the pVAX-RBD construct $(p<0.01)$ and 10000 times higher than in the control group which received pVAX-PGS $(p<0.01)$ (Fig. 5b).

Virus neutralization activity of the sera was assessed in the in vitro neutralization assay using live SARS-CoV-2 virus. The blood sera from the mice immunized with pVAX-RBD-PGS were able to neu-

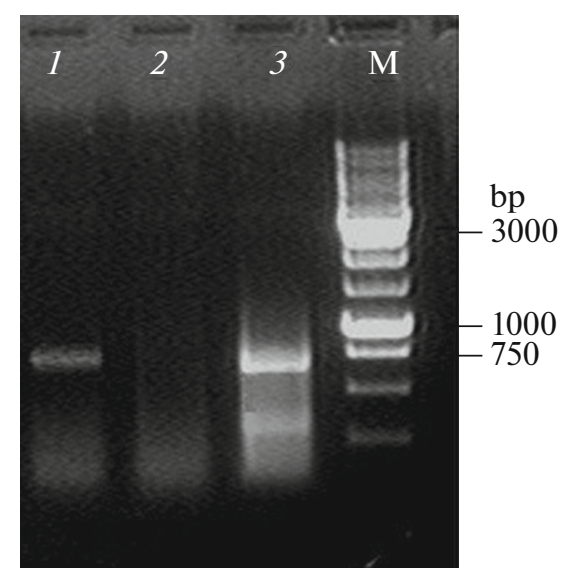

Fig. 2. Electrophoretic analysis of the RT-PCR products in $1 \%$ agarose gel. Lanes (1) and (2) PCR products obtained using total RNA from HEK-293T cells transfected with pVAX-RBD and pVAX, respectively; lane (3) PCR products obtained using the RBD-encoding pVAXRBD plasmid as a positive control. M-M12 DNA ladder (SibEnzyme, Russia). 


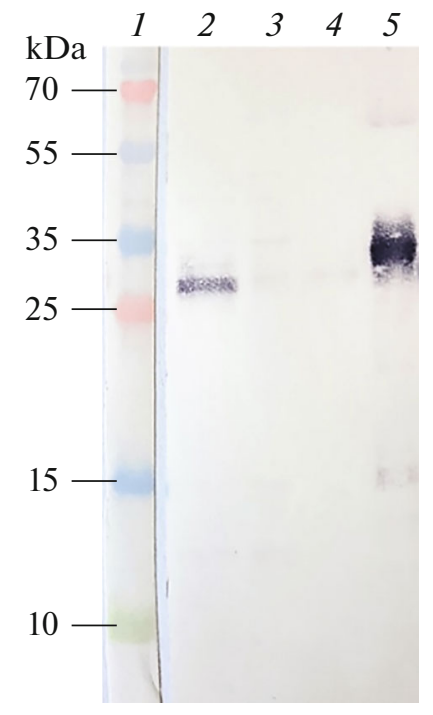

Fig. 3. Analysis of RBD expression in the CHO-K1 cells transfected with pVAX-RBD and pVAX. Lane (1) protein molecular weight marker; (2) and (3) cell lysates obtained from the CHO-K1 cells transfected with pVAX-RBD and pVAX, respectively; (4) and (5) cultural liquid obtained from the CHO-K1 cells transfected with pVAX and pVAX$\mathrm{RBD}$, respectively.

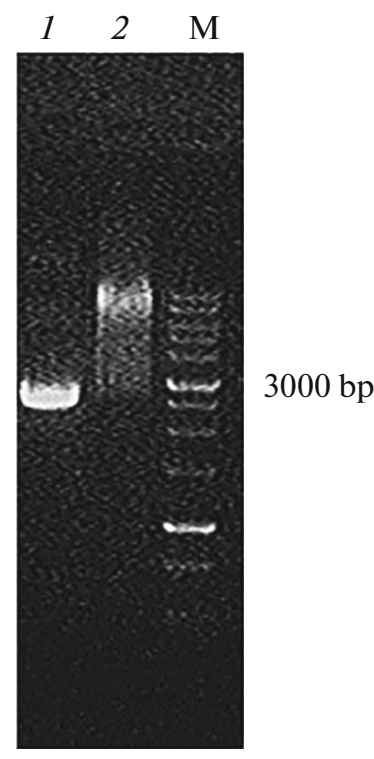

Fig. 4. Analysis of the pVAX-RBD-PGS complex formation. The results of $1 \%$ agarose gel electrophoresis are presented, DNA : PGS ratio is $1: 10$. Lane (1) pVAX-RBD plasmid and lane (2) pVAX-RBD plasmid-PGS complex, M-M12 DNA ladder (SibEnzyme).

tralize the $\mathrm{nCoV} /$ Victoria/1/2020 SARS-CoV-2 strain at a $1: 200$ dilution, while the sera from the group immunized with pVAX-RBD, at a $1: 12$ dilution $(p<$ $0.01)$. The sera from the control animals showed no neutralizing activity (Fig. 5c).
pVAX-RBD Induces Cellular Immune Response

The T-cell immune response was assessed based on the number of IFN- $\gamma$-producing splenocytes using the ELISpot technique. Splenocytes were stimulated with the pool of S protein peptides.

It has been demonstrated that the splenocytes isolated from mice spleens two weeks after the third immunization with the PGS-enveloped and nonenveloped pVAX-RBD constructs exhibited stronger IFN- $\gamma$ response to the stimulation with virus peptides than the splenocytes isolated from the control group $(p<0.01)$ (Fig. 6). Hence, a conclusion could be made that the immunized animals developed the RBD-specific T-cell response. The level of IFN- $\gamma$-producing $\mathrm{T}$-lymphocytes was slightly higher in the group of animals which received pVAX-RBD-PGS compared to the group which received the "naked" pVAX-RBD DNA, these differences being statistically insignificant.

\section{DISCUSSION}

DNA vaccines are one of the platforms which have been used to develop candidate vaccines against COVID-19, a number of them are currently undergoing phase III clinical trials. Such vaccines may be easily produced after the nucleotide sequence of virus genome has been published. The production of an antigen in its native conformation, the activation of the humoral and cellular immunity, easy manufacturing, low production costs, no specific storage requirements, and safety are among the advantages of DNA vaccines.

One of the main drawbacks of DNA vaccines is their low immunogenicity of "naked" plasmid DNA administered intramuscularly [9]. To increase the efficiency of DNA vaccine delivery to antigen-presenting cells, cationic polymers, proteins, and lipids are frequently used allowing neutralization of the negative charge of the nucleic acid thereby facilitating its transport across the cell membrane of the antigen-presenting cells. Needle-free injectors and electroporators may also be used [19-23].

For example, Smith et all. [8] used electroporation to immunize mice with a DNA construct encoding the full-size $\mathrm{S}$ protein. A single dose containing as little as $2.5 \mu \mathrm{g}$ of DNA induced a humoral immune response (antibody titer $1: 1000)$ and T-cell response (1000 IFN- $\gamma$-producing cells per $10^{6}$ splenocytes). Seo et al. [24] also designed a DNA construct encoding the $\mathrm{S}$ protein and the N-terminal part of the tissue plasminogen activator as a leader sequence. Two doses of this DNA construct $(50 \mu \mathrm{g})$ administered to mice using electroporation resulted in a strong specific response with the antibody titers of $1: 10000$, with the number of IFN- $\gamma$-producing T-lymphocytes reaching 2000 per $10^{6}$ splenocytes in certain cases. Prompetchara et al. [25] also studied the immunogenic properties of the DNA constructs encoding the full-size $\mathrm{S}$ protein 
(a)

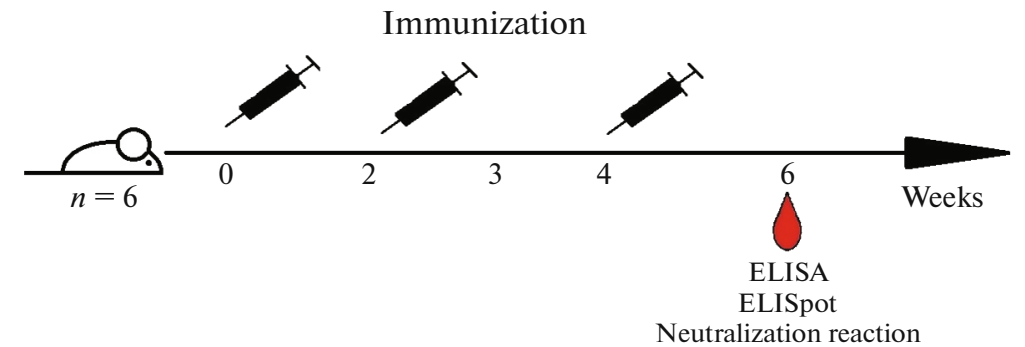

(b)

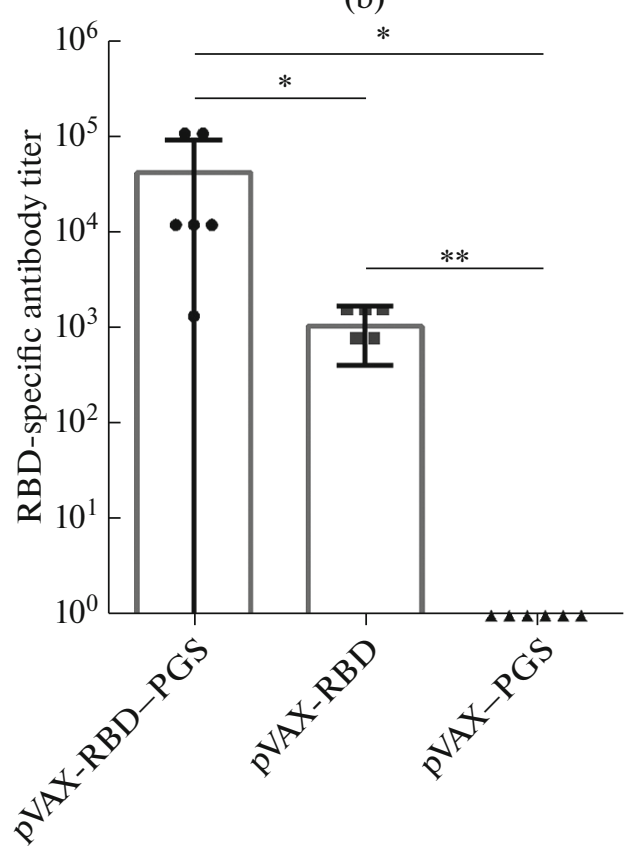

Neutralization reaction

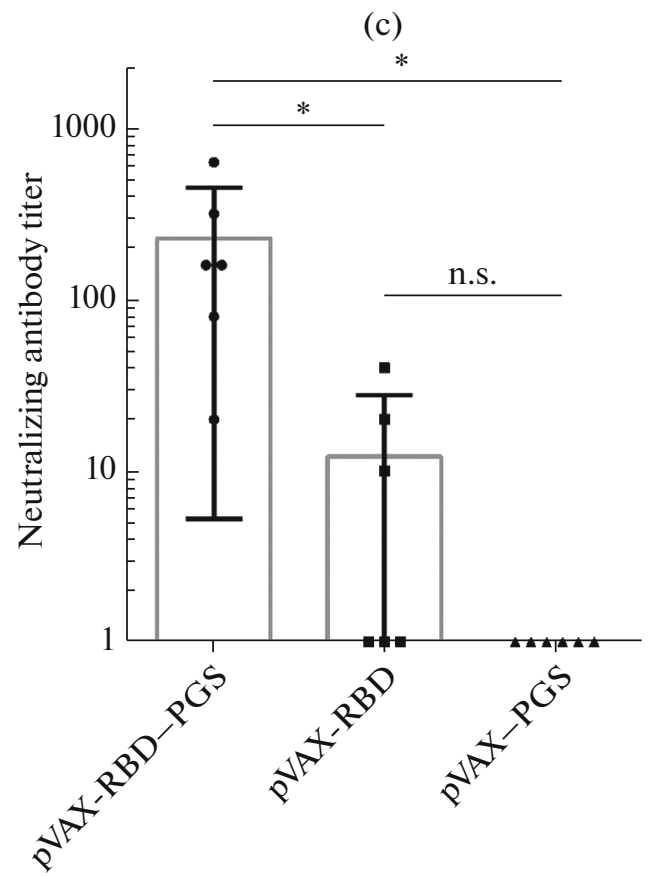

Fig. 5. Humoral immune response induced by the pVAX-RBD-PGS, pVAX-RBD, and pVAX-PGS constructs in mice. (a) Immunization and sample collection timeline: vaccine constructs were administered to mice three times in two-week intervals. Six weeks after the first immunization, blood and spleens were collected from mice for analysis. (b) RBD-specific IgG antibody titers were assessed in ELISA. Reciprocal titer (dilution) values are shown in the plot. (c) Virus neutralization activity of sera was assessed using the SARS-CoV-2 (nCoV/Victoria/1/2020 strain) neutralization reaction. Reciprocal titer values are provided in the plot. Statistical data processing was carried out using GraphPad Prism 6.0 software. Data are presented as mean \pm standard deviation (SD). Statistical significance was assessed using the nonparametric Mann-Whitney test (n.s. - no statistical significance, ${ }^{*} p<0.01$, and $\left.{ }^{*} p<0.05\right)$.

or two of its domains S1 and S2. Immunization was carried out in three doses using electroporator, each DNA dose amounting to $100 \mu \mathrm{g}$. The administration of the studied constructs resulted in the production of specific antibodies (with a titer of $1: 2000)$ and high $\mathrm{T}$-cell response indices, especially in the case of the DNA construct encoding the full-size $\mathrm{S}$ protein (3000 IFN- $\gamma$-producing cells per $10^{6}$ splenocytes). A group of Japanese researchers, Nishikawa et al. [26], employed the Pyro-drive Jet injector to subcutaneously administer the candidate DNA vaccine to mice. After the administration of the three doses of the construct, the authors observed high levels of specific antibodies with the titers equaling $1: 10000$. At the same time, the T-cell response was weaker than in the case of vaccine construct administration using elec- troporation (150 IFN- $\gamma$-producing cells per $10^{6}$ splenocytes vs $1000-3000$ in the case of electroporation) [26]. To summarize, it should be noted that using electroporation to administer vaccine DNA constructs to the animals promotes the development of both humoral and cellular immune response to the target antigen. Among the shortcoming of this technique are pain in the injection site and the need for specific equipment which have not yet been certified for human use.

Currently, a number of companies are developing DNA vaccines against COVID-19. Vaccines produced by Inovio, AnGes, and Zydus Cadila are currently at the start of phase III clinical trials (https://www.who.int/ publications/m/item/draft-landscape-of-covid-19-candidate-vaccines). Eight more vaccines are at the earlier stages of clinical trials. 


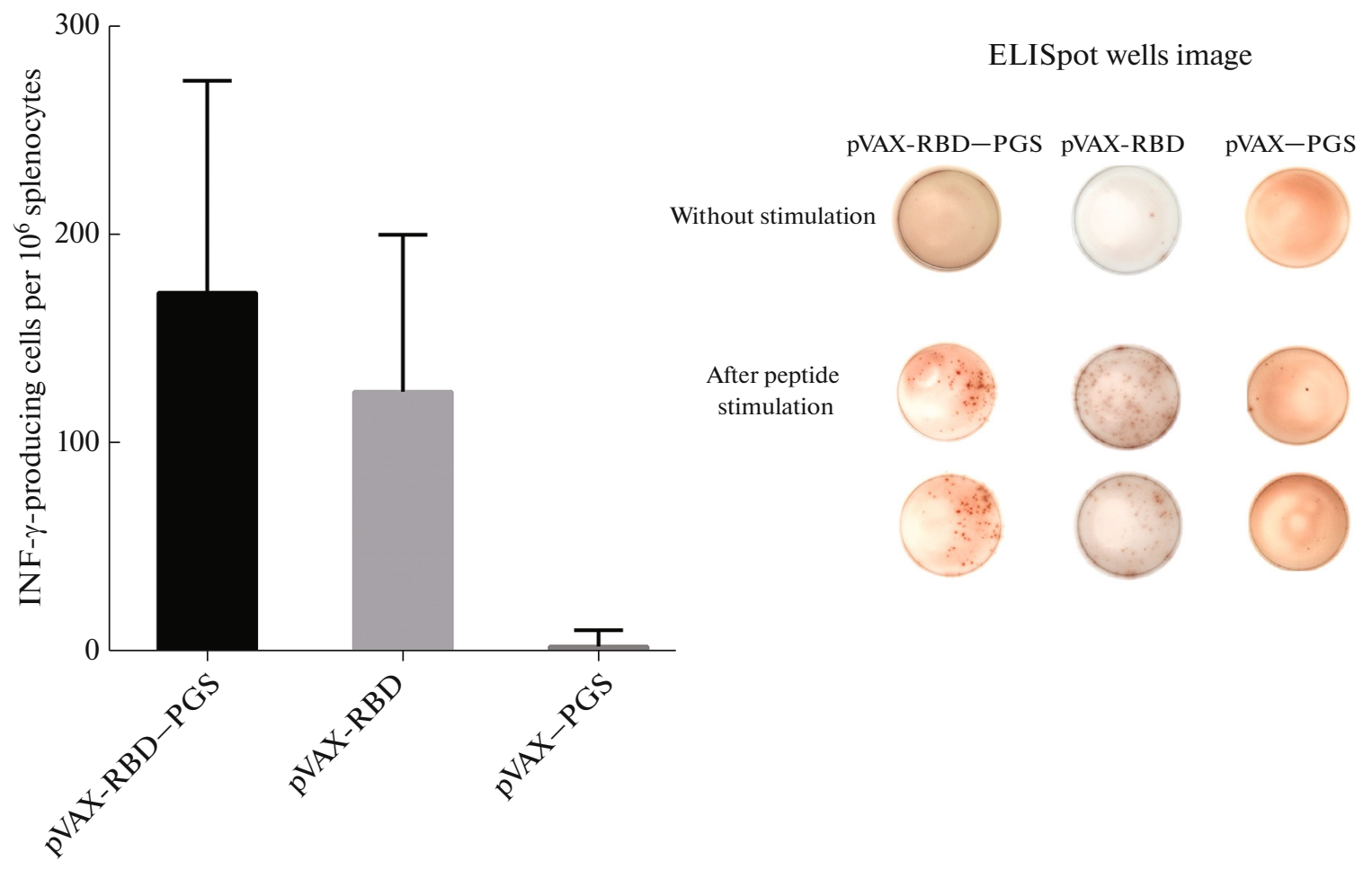

Fig. 6. Comparative study of the T-cell response in mice immunized with the pVAX-RBD-PGS, pVAX-RBD, and pVAX-PGS. The number of IFN- $\gamma$-producing cells was determined using the ELISpot technique. The differences between the groups immunized with the vaccine constructs and the control plasmid were statistically significant $(p<0.01)$, while the differences between the groups immunized with pVAX-RBD-PGS and pVAX-RBD were statistically insignificant. Statistical data processing was carried out using the GraphPad Prism 6.0 software. Data are presented as mean \pm standard deviation (SD). Statistical significance was assessed using the nonparametric Mann-Whitney test.

For one of the ten DNA vaccines currently in clinical trials a liposome-based delivery system was used, while most of the vaccines are suggested to be administered using specific equipment (https://clinicaltrials.gov/ct2/show/NCT04591184). Towards this end, the Fusogenix platform has been specifically designed, which represents a mixture of neutral lipids and small membrane proteins involved in membrane fusion. Although the toxicity of such liposomes appears to be significantly lower, they are still not devoid of certain important drawbacks both related to the liposomes themselves and the technically challenging transportation and storage of such vaccines which require cold chain maintenance [16, 27].

We have suggested a new means of DNA vaccine delivery consisting of packaging the nucleic acid into the polycationic polyglucine-spermidine conjugate which we refer to as PGS. Our choice was based on the previous results obtained when testing PGS as a packaging means to deliver nucleic acid-based vaccine constructs into the cells [14-16]. PGS is a component of CombiHIVvac, a candidate vaccine against HIV, which has already completed phase I clinical trials [14]. PGS-containing DNA preparations may be lyophilized (which makes them quite different from the liposome-containing preparations) and stored at $4{ }^{\circ} \mathrm{C}$ for at least two years without losing activity. Importantly, PGS components are biodegradable and safe for humans. Being cheap, safe, and allowing lyophilization with subsequent long storage, they give additional technological advantages when it comes to vaccine manufacturing and transportation.

It is well-known that DNA vaccines are usually more efficient at inducing the $\mathrm{T}$-cell response than the B-cell response because the antigens encoded by a DNA vaccine are synthesized and processed inside the cell, intracellular proteins being present bound with MHC class I molecules, which induce the activation of the cytotoxic $\mathrm{T}$ lymphocytes with activity directed against virus-infected cells. To successfully protect against SARS-CoV-2, a vaccine should induce both the cellular and humoral immune response [28]. Taking this into consideration, we supposed that including the leader sequence 176 combining the signal sequences of two actively secreted proteins into the immunogene may promote efficient RBD secretion from the cell and hence, enhance the humoral immune response to the DNA construct used.

The ability of the designed DNA-vaccine to promote the target protein secretion from eukaryotic cells 
was assessed using the cultural liquid from the pVAXRBD-transfected CHO-K1 cells (Fig. 3).

To obtain the DNA-PGS complexes, the pVAXRBD plasmid and the PGS conjugate was mixed in a $1: 10$ weight ratio, with complex formation being monitored using electrophoresis in $1 \%$ agarose gel (Fig. 4).

Comparative immunogenicity study of the "naked" plasmid, pVAX-RBD, and the PGS-enveloped pVAXRBD plasmid, pVAX-RBD-PGS, demonstrated the latter to be more efficient in inducing the immune response. ELISA revealed that the RBD-specific antibody titers in the blood sera of animals in the pVAXRBD group equaled $1: 1000$, while it was $1: 42000$ in the pVAX-RBD-PGS group (Figs. 5b, 5c). Both nonenveloped and PGS-enveloped pVAX-RBD constructs also efficiently induced the cellular immune response in the animals. According to the ELISpot results, the splenocytes of the immunized animals efficiently produced INF- $\gamma$ in response to stimulation with the SARS-CoV-2 S protein peptide pool (Fig. 6).

To summarize, the pVAX-RBD construct coated with the polyglucine-spermidine conjugate may be considered as a promising candidate DNA vaccine against COVID-19 with its immunological properties being comparable with those of the vaccine DNA constructs tested in mice $[8,24-26]$.

\section{FUNDING}

The work was supported by the Ministry of Science and Higher Education of the Russian Federation (grant no. 07515-2019-1665).

\section{COMPLIANCE WITH ETHICAL STANDARDS}

Conflict of interest. The authors declare they have no conflict of interest.

All studies involving animals were carried out in accordance with the "Guidelines for experimentation using laboratory animals". All experiments were approved by the Bioethical Committee of the Vector State Research Center of Virology and Biotechnology, Russian Federal State Agency for Health and Consumer Rights Surveillance, protocol no. GNTs VB Vector/10-09.2020.

\section{REFERENCES}

1. Gao Q., Bao L., Mao H., Wang L., Xu K., Yang M., Li Y., Zhu L., Wang N., Lv Z., Gao H., Ge X., Kan B., Hu Y., Liu J., et al. 2020. Development of an inactivated vaccine candidate for SARS-CoV-2. Science. 369, 77-81.

2. Wang H., Zhang Y., Huang B., Deng W., Quan Y., Wang W., Xu W., Zhao Y., Li N., Zhang J., Liang H., Bao L., Xu Y., Ding L., Zhou W., et al. 2020. Development of an inactivated vaccine candidate, BBIBP-CorV, with potent protection against SARS-CoV-2. Cell. 182, $39-51$.

3. Keech C., Albert G., Cho I., Robertson A., Reed P., Neal S., Plested J.S., Zhu M., Cloney-Clark S., Zhou H.,
Smith G., Patel N., Frieman M.B., Haupt R.E., Logue J., et al. 2020. Phase 1-2 trial of a SARS-CoV-2 recombinant spike protein nanoparticle vaccine. N. Engl. J. Med. 383, 2320-2332.

4. Ravichandran S., Coyle E.M., Klenow L., Tang J., Grubbs G., Liu S., Wang T., Golding H., Khurana S. 2020. Antibody signature induced by SARS-CoV-2 spike protein immunogens in rabbits. Sci. Transl. Med. 12, eabc3539.

5. Quinlan B.D., He W., Mou H., Zhang L., Guo Y., Chang J., Peng S., Ojha A., Tavora R., Parcells M.S., Luo G., Li W., Zhong G., Choe H., Farzan M. 2020. An engineered receptor-binding domain improves the immunogenicity of multivalent SARS-CoV-2 vaccines. bioRxiv. 2020.11.18.388934.

https://doi.org/10.1101/2020.11.18.388934

6. García-Arriaza J., Garaigorta U., Pérez P., LázaroFrías A., Zamora C., Gastaminza P., Del Fresno C., Casasnovas J.M., Sorzano C.Ó.S., Sancho D., Esteban M. 2021. COVID-19 vaccine candidates based on modified vaccinia virus Ankara expressing the SARS-CoV-2 spike induce robust T- and B-cell immune responses and full efficacy in mice. J. Virol. 95 (7), e02260-20. https://doi.org/10.1128/JVI.02260-20

7. Logunov D.Y., Dolzhikova I.V., Zubkova O.V., Tukhvatulin A.I., Shcheblyakov D.V., Dzharullaeva A.S., Grousova D.M., Erokhova A.S., Kovyrshina A.V., Botikov A.G., Izhaeva F.M., Popova O., Ozharovskaya T.A., Esmagambetov I.B., Favorskaya I.A., et al. 2020. Safety and immunogenicity of an rAd26 and rAd5 vectorbased heterologous prime-boost COVID-19 vaccine in two formulations: Two open, non-randomised phase $1 / 2$ studies from Russia. Lancet. 396, 887-897.

8. Smith T.R.F., Patel A., Ramos S., Elwood D., Zhu X., Yan J., Gary E.N., Walker S.N., Schultheis K., Purwar M., Xu Z., Walters J., Bhojnagarwala P., Yang M., Chokkalingam N., et al. 2020. Immunogenicity of a DNA vaccine candidate for COVID-19. Nat. Commun. 11, 2601.

9. Yu J., Tostanoski L.H., Peter L., Mercado N.B., McMahan K., Mahrokhian S.H., Nkolola J.P., Liu J., Li Z., Chandrashekar A., Martinez D.R., Loos C., Atyeo C., Fischinger S., Burke J.S., et al. 2020. DNA vaccine protection against SARS-CoV-2 in rhesus macaques. Science. 369, 806-811.

10. Walsh E.E., Frenck R.W. Jr, Falsey A.R., Kitchin N., Absalon J., Gurtman A., Lockhart S., Neuzil K., Mulligan M.J., Bailey R., Swanson K.A., Li P., Koury K., Kalina W., et al. 2020. Safety and immunogenicity of two RNA-based Covid-19 vaccine candidates. N. Engl. J. Med. 383, 2439-2450.

11. Baden L.R., El Sahly H.M., Essink B., Kotloff K., Frey S., Novak R., Diemert D., Spector S.A., Rouphael N., Creech C.B., McGettigan J., Khetan S., Segall N., Solis J., Brosz A., et al. 2021. Efficacy and safety of the mRNA-1273 SARS-CoV-2 vaccine. N. Engl. J. Med. 384, 403-416.

12. Verdecchia P., Cavallini C., Spanevello A., Angeli F. 2020. The pivotal link between ACE2 deficiency and SARS-CoV-2 infection. Eur. J. Intern. Med. 76, 14-20.

13. Karpenko L.I., Lebedev L.R., Bazhan S.I., Korneev D.V., Zaitsev B.B., Ilyichev A.A. 2017. Visualization of CombiHIVvac vaccine particles using electron microscopy. AIDS Res. Hum. Retroviruses. 33, 323-324. 
14. Karpenko L.I., Bazhan S.I., Bogryantseva M.P., Ryndyuk N.N., Ginko Z.I., Kuzubov V.I., Lebedev L.R., Kaplina O.N., Reguzova A.Yu., Ryzhikov A.B., Usova S.V., Oreshkova S.F., Nechaeva E.A., Danilenko E.D., Ilyichev A.A. 2016. Results of phase I clinical trials of a combined vaccine against HIV-1 based on synthetic polyepitope immunogens. Russ. J. Bioorg. Chem. 42 (2), $170-182$.

15. Karpenko L.I., Apartsin E.K., Dudko S.G., Starostina E.V., Kaplina O.N., Antonets D.V., Volosnikova E.A., Zaitsev B.N., Bakulina A.Y., Venyaminova A.G., Ilyichev A.A., Bazhan S.I. 2020. Cationic polymers for the delivery of the Ebola DNA vaccine encoding artificial T-cell immunogen. Vaccines. 8, 718.

16. Karpenko L.I., Rudometov A.P., Sharabrin S.V., Shcherbakov D.N., Borgoyakova M.B., Bazhan S.I., Volosnikova E.A., Rudometova N.B., Orlova L.A., Pyshnaya I.A., Zaitsev B.N., Volkova N.V., Azaev M.S., Zaykovskaya A.V., Pyankov O.V., Ilyichev A.A. 2020. Delivery of mRNA vaccine against SARS-CoV-2 using a polyglucin:spermidine conjugate. Vaccines. 9, 76.

17. Reed L.J., Muench H. 1938. A simple method of estimating fifty per cent endpoints. Am. J. Hyg. 27 (3), 493-497. https://doi.org/10.1093/oxfordjournals.aje.a118408

18. Brito L.A., Singh M. 2011. Acceptable levels of endotoxin in vaccine formulations during preclinical research. J. Pharm. Sci. 100, 34-37.

19. Milani A., Bolhassani A., Heshmati M. 2017. Delivery of HIV-1 Nef linked to heat shock protein 27 using a cationic polymer is more effective than cationic lipid in mammalian cells. Bratisl. Lek. Listy. 118, 334-338.

20. Saljoughian N., Zahedifard F., Doroud D., Doustdari F., Vasei M., Papadopoulou B., Rafati S. 2013. Cationic solid-lipid nanoparticles are as efficient as electroporation in DNA vaccination against visceral leishmaniasis in mice. Parasite Immunol. 35, 397-408.

21. Jiang J. 2021. Cell-penetrating peptide-mediated nanovaccine delivery. Curr. Drug Targets. 22 (8), 896-912. https://doi.org/10.2174/1389450122666210203193225
22. Akhtar N., Singh V., Yusuf M., Khan R.A. 2020. Noninvasive drug delivery technology: Development and current status of transdermal drug delivery devices, techniques and biomedical applications. Biomed Tech. 65, 243-272.

23. Tebas P., Yang S., Boyer J.D., Reuschel E.L., Patel A., Christensen-Quick A., Andrade V.M., Morrow M.P., Kraynyak K., Agnes J., Purwar M., Sylvester A., Pawlicki J., Gillespie E., Maricic I., et al. 2021. Safety and immunogenicity of INO-4800 DNA vaccine against SARS-CoV-2: A preliminary report of an open-label, phase 1 clinical trial. EClinicalMedicine. 31, 100689. https://doi.org/10.1016/j.eclinm.2020.100689

24. Seo Y.B., Suh Y.S., Ryu J.I., Jang H., Oh H., Koo B.S., Seo S.H., Hong J.J., Song M., Kim S.J., Sung Y.C. 2021. Soluble spike DNA vaccine provides long-term protective immunity against SARS-CoV-2 in mice and nonhuman primates. Vaccines. 9, 307.

25. Prompetchara E., Ketloy C., Tharakhet K., Kaewpang P., Buranapraditkun S., Techawiwattanaboon T., SatheanAnan-Kun S., Pitakpolrat P., Watcharaplueksadee S., Phumiamorn S., Wijagkanalan W., Patarakul K., Palaga T., Ruxrungtham K. 2021. DNA vaccine candidate encoding SARS-CoV-2 spike proteins elicited potent humoral and Th1 cell-mediated immune responses in mice. PLoS One. 16, e0248007.

26. Nishikawa T., Chang C.Y., Tai J.A., Hayashi H., Sun J., Torii S., Ono C., Matsuura Y., Ide R., Mineno J., Sasai M., Yamamoto M., Nakagami H., Yamashita K. 2021. Anti-CoVid19 plasmid DNA vaccine induces a potent immune response in rodents by Pyro-drive Jet Injector intradermal inoculation. bioRxiv. https://doi.org/10.1101/2021.01.13.426436

27. Chang H.I., Yeh M.K. 2012. Clinical development of liposome-based drugs: Formulation, characterization, and therapeutic efficacy. Int. J. Nanomed. 7, 49-60.

28. Lagunas-Rangel F.A, Chávez-Valencia V. 2021. What do we know about the antibody responses to SARSCoV-2? Immunobiology. 226, 152054.

Translated by E. Martynova 